Cite this: RSC Adv., 2014, 4, 10334

Received 12th December 2013 Accepted 1st February 2014

DOI: $10.1039 / c 3 r a 47574 c$

www.rsc.org/advances

\title{
Silicon nanocrystals and nanodiamonds in live cells: photoluminescence characteristics, cytotoxicity and interaction with cell cytoskeleton $\dagger$
}

\author{
A. Fucikova, ${ }^{\text {*a }}$ J. Valenta, ${ }^{\text {I I. Pelant, }}{ }^{\text {b }}$ M. Hubalek Kalbacova, ${ }^{c}$ A. Broz, ${ }^{c}$ B. Rezek, ${ }^{\text {b }}$ \\ A. Kromkab and Z. Bakaeva ${ }^{d}$
}

\begin{abstract}
The number of newly developed nanomaterials is steadily increasing but only a few are suitable for applications in biology. These nanomaterials are often made from harmful compounds and there is no convenient technique to remove them from the body after application. This study is focused on silicon nanocrystals and nanodiamonds, which are two promising nanomaterials for bio-applications. Silicon nanocrystals (Si-NCs) are exceptional since they provide several desired properties: low cytotoxicity, suitability for chemical activation, efficient photoluminescence, and bio-degradability. All these parameters promote application of Si-NCs in living organisms and even human medicine. Nanodiamonds (NDs), on the other hand, are non-biodegradable which limits their use to mostly long term in vitro studies. However, when using these nanomaterials one needs to address the effect of accumulation and aggregation of such materials in cells and how it contributes to the overall cytotoxicity. Here, we present studies on the interaction of two promising nanomaterials Si-NCs and NDs with actin structure of mammalian cells, evaluation of their cytotoxicity by various methods, and observation of single nanoparticle luminescence spectra within living cells. According to our results $\mathrm{Si}-\mathrm{NCs}$ are more promising for application in nanomedicine compared to NDs.
\end{abstract}

\section{Introduction}

A great variety of nanomaterials have been developed in recent years. Their application ranges from coatings and functional structures $^{\mathbf{1 , 2}}$ in many consumer products to drug carriers or even food additives., ${ }^{2,3}$ Despite these expanding and intense uses, long term studies on the impact of nanomaterials on humans and comparative studies between individual nanomaterials are still rare. In this work, we concentrate on two nanomaterials: mainly silicon nanocrystals (Si-NCs) and secondly diamond nanocrystals (NDs). They are studied in the perspective of their use as novel fluorescent markers in biology, and, possibly (in case of Si-NCs), in human medicine and other biological disciplines. In particular, Si-NCs hold great promise

${ }^{a}$ Department of Chemical Physics and Optics, Faculty of Mathematics and Physics, Charles University, Prague 2, CZ-121 16, Czech Republic. E-mail: anna.fucikova@ email.cz

${ }^{5}$ Institute of Physics AS CR, v. v. i., Prague 6, CZ-162 53, Czech Republic

'Institute of Inherited Metabolic Disorders, 1 st Faculty of Medicine, Charles University, Prague, CZ-128 53, Czech Republic

${ }^{d}$ Institute of Macromolecular Chemistry AV CR, v. v. i., Prague 6, CZ-162 06, Czech Republic

$\uparrow$ Electronic supplementary information (ESI) available: Growth curves of HeLa cells incubated with A-Si-NC, CQD, A-ND, or ND and of a control sample incubated without added nanomaterials, high resolution of Fig. 5, Z-cross section of the cells. See DOI: 10.1039/c3ra47574c in substituting artificial stains and harmful quantum dots (few nanometer sized particles with photoluminescence abilities, e.g. CdSe), which are currently used in laboratories worldwide as imaging and tracking tools in microscopy.

The first quantum dots (QDs) used in biology were based on CdSe (discovered in the $1980 \mathrm{~s}^{4}$ ). The primary bio-conjugation study on CdSe QDs was performed around the year 2000 (ref. 5) and adequate biological study was carried out in $2004 .^{6}$ Initially, they were assumed to be biologically safe, but studies in 2008 and later revealed their considerable toxicity.,8 From the discovery of CdSe QD it took almost 30 years until they were proven cytotoxic. Silicon nanocrystals ${ }^{9}$ and nanodiamonds ${ }^{\mathbf{1 0}}$ (both discovered around 1990) are supposed to from the next generation of QDs, and until now, they have not shown significant cytotoxicity. One of the first biological articles studying interaction between Si-NCs and cells was published in 2008 (ref. 11) and in the case of ND in 2007. ${ }^{12}$

As opposed to commonly used inorganic- and protein-based fluorescent dyes, Si-NCs and NDs are quite stable in a biological environment. Even more, NDs are highly resistant to physical degradation and remain intact to chemicals. ${ }^{13}$ However, there is no known pathway to remove them from living organisms (or even from single cells), and ND biocompatibility or cytotoxicity $^{\mathbf{1 4 - 1 8}}$ are questionable when accumulated to larger volumes as discussed below in this article. On the other hand, Si-NCs are biodegradable in a living organism ${ }^{19-21}$ and the 
process of biodegradation is not the subject of this study. Furthermore, silicon is, according to medical reports, important for the development of human bones and body functionality $^{22}$ and might be used even as a food additive. ${ }^{3}$ The pathway of removing Si-NCs from body is based on their slow conversion into silicic acids. ${ }^{23}$

The studies on Si-NCs are slowly moving to real applications in medicine and biology. First study on single-molecule tracking and fluorescence imaging in cell with use of Si-NCs was recently published in. ${ }^{24}$ First studies on the attachment of DNA to Si-NC surface $^{25}$ are opening up possibilities for precise addressing of SiNCs in biological environment. Recently two studies have been published on in vivo toxicity of Si-NCs in mice and monkeys. ${ }^{26,27}$ Their results show possibility of using Si-NCs in human medicine.

In this article, we address the issue of the safe dose and related cytotoxicity of luminescent $\mathrm{Si}$-NCs and ND nanoparticles in mammalian cells. Studying cytotoxicity of nanoparticles is more complicated compared to drugs since nanoparticles can interfere with reagents from the cytotoxicity test and produce unreliable results. ${ }^{28}$ For this reason, various cytotoxicity assays testing different cell processes should be performed at the same incubation time. The cytotoxicity test may also produce different results depending on the growth phase of cells (freshly plated or already attached to surface, as discussed in the article) and how nanomaterial is introduced into the cell culture.,19

The estimations of desired dose for future use in biology are: for single dot tracking in the cell, the dose might be as low as few nanoparticles per cell; for use as fluorescent marker in biology, up to $1 / 10$ of the maximal dose used in this article and for use as drug carrier on the same level or even higher than the maximal dose used in this study.

The smallest Si-NCs used in this study are sized around 2.5$3 \mathrm{~nm}$ in diameter and NDs around $8 \mathrm{~nm}$ (larger NDs are usually used in literature: $25 \mathrm{~nm},{ }^{29} 50 \mathrm{~nm}$ (ref. 30)). Mixtures of variously sized aggregates (up to $460 \mathrm{~nm}$ ) of individual nanoparticles were used to simulate possible aggregation effects in living organisms. The study of aggregation and accumulation of nanoparticles within living cells is an important issue, because overall cytotoxicity may increase dramatically. Cytotoxic and biological studies are still rare for both Si-NCs and NDs and comparative studies between these two outstanding nanomaterials are completely missing.

\section{Materials and methods}

\section{Si-NCs preparation}

Si-NCs were obtained in the form of porous silicon by electrochemical etching of p-type [100] Si boron doped wafers with resistivity $0.075-0.100 \Omega \mathrm{cm}$. The etching solution consisted of hydrofluoric acid, hydrogen peroxide, and ethanol. The etching current density was $2.6 \mathrm{~mA} \mathrm{~cm}{ }^{-2}$. Once the etching completed, the wafer covered with porous silicon was treated in hydrogen peroxide for 5-20 minutes to obtain finer porosity. Powder of Si-NCs was obtained by mechanical scratching of the porous layer from the substrate. The resulting size of Si-NCs ranges between 1 and $4 \mathrm{~nm}$ (DLS) depending on etching time and peroxide treatment. The $\mathrm{Si}-\mathrm{NC}$ is made of Si crystalline core covered with $\mathrm{SiO}_{2}$ layer (for detailed high-resolution transmission electron microscopy (HRTEM) image see ref. 31).

\section{ND preparation}

NDs were supplied by NanoCarbon UDD-TAH, Diamond Centre, St. Petersburg, Russia. NDs were prepared by combustion of oxygen-deficient explosive mixture of TNT/T4 in a closed chamber and afterwards purified in various acids. ${ }^{32}$ The ND surface is mainly covered with carboxyl groups but also various ketone, hydroxyl and oxygen groups are present on the surface. ${ }^{33}$ The product consisted of a mixture of ND particles sized 8-10 nm (56.6 vol\%) and $460 \mathrm{~nm}$ (43.4 vol\%).

\section{Atomic force microscope (AFM) measurement}

The AFM images of Si-NCs were obtained by the Agilent Technologies 5500 AFM with a multipurpose small scanner $(9 \mu \mathrm{m} x y)$ in AAC mode (air). The Si-NC/ethanol colloids were dropped onto a highly-ordered pyrolytic graphite and dried. Morphology measurements of NDs were carried out in a Veeco DI 3100 IV atomic force microscope in tapping mode using Multi75Al cantilevers. The measurements were repeated with ICON AFM in PeakForce QNM mode with similar results.

\section{Dynamic light scattering (DLS) and zeta-potential measurements}

Dynamic light scattering of nanomaterial colloidal suspension in water was measured with Zetasizer Nano S (Malvern). The colloidal sample was illuminated by a He-Ne laser, $633 \mathrm{~nm}$, $4 \mathrm{~mW}$ and scattered signal detected with an avalanche photodiode in backscattering geometry $173^{\circ}$ (easier characterization of small particles). The zeta potential was measured with a Zetasizer Nano Z (Malvern). The data were processed with Zetasizer Software. Samples were measured in water and nutrient media with and without bovine serum.

\section{Growth curves}

Growth curves were measured for both L929 (mouse fibroblast) and HeLa (human cervical carcinoma) cells. Cells were incubated under standard conditions $\left(5 \% \mathrm{CO}_{2}\right.$, at $\left.37^{\circ} \mathrm{C}\right)$, in nutrient media for L929 cells consisting of: MEM 92\%, L-glutamine 1\%, antibiotics/antimycotic $1 \%$, fetal calf serum $5 \%, \mathrm{NaHCO}_{3} 1 \%$ (all from PAA); for HeLa cells: EMEM 86\%, L-glutamine 1\%, antibiotics/antimycotic 1\%, fetal calf serum $10 \%, \mathrm{NaHCO}_{3} 1 \%$, nonessential amino acids 1\% (all from PAA) with the studied nanomaterials (nanomaterials were added immediately after the passage of cells) for about 80 hours and the number of living and dead cells was counted in each sample in 8 hour intervals; each experiment was repeated 12 times. A growth curve represents the number of living cells (observed within a field of view of a transmission optical microscope (Olympus IX 51) with $20 \times$ objective - approximately $0.5 \mathrm{~mm}^{2}$ ) plotted against time.

\section{SAOS-2 cells cultivation and transfection for live cell imaging}

The SAOS-2 human osteosarcoma cells (osteoblast-like cell line) were standardly held in cell incubator with $5 \% \mathrm{CO}_{2}$, at $37{ }^{\circ} \mathrm{C}$, in 
nutrient media consisting of McCoy's 5A (Bioconcept) with L-glutamine, 15\% fetal bovine serum (PAA), Penicillin 20 units per $\mathrm{ml}$ and Streptomycin $20 \mathrm{mg} \mathrm{ml}^{-1}$ (Sigma Aldrich). To obtain the cells with fluorescently tagged actin for live cell fluorescence microscopy the SAOS-2 cells were stably transfected by pEYFPN1 plasmid with inserted gene for actin. The cells then continuously expressed yellow-stained actin. The transfection was performed using the transfection reagent Lipofectamine (Invitrogen) according to the manufacturer's protocol at standard cultivation conditions $\left(37^{\circ} \mathrm{C}, 5 \% \mathrm{CO}_{2}\right)$ under the $\mathrm{G} 418$ selection.

\section{SEM images of HeLa cells incubated with Si-NCs and ND}

The samples were measured with JEOL JSM-6300 apparatus using accelerating voltage $5 \mathrm{kV}$, image detector Tescan $2048 \times$ $1536 \times 8$ bit, vacuum 10-4 Pa; resolving power under these conditions was $11 \mathrm{~nm}$. Nanomaterials were added 6 hours after the cells were plated and incubated in standard nutrient media for HeLa cells (EMEM 86\%, newborn calf serum 10\%, antibiotics antimicotics 1\%, non-essential amino acids 1\%, L-glutamine $1 \%, \mathrm{NaHCO}_{3} 1 \%$ ). After addition of nanomaterials the cells were incubated for another 18 hours. The amount of nanomaterials was $4 \mu \mathrm{g} \mathrm{ml}^{-1}$ for Si-NCs and $10 \mu \mathrm{g} \mathrm{ml}^{-1}$ of NDs (the different concentrations were chosen in order to have approximately the same number of particles in the sample). After incubation, the cells were washed with phosphate buffered salt, fixed by glutaraldehyde fixative, drained and gilded.

\section{Live cell imaging}

Live cell imaging was performed using microscope Nikon TE2000-E equipped with a cultivation chamber which enables temperature and atmosphere control $\left(37{ }^{\circ} \mathrm{C}, 5 \% \mathrm{CO}_{2}\right)$. The confocal images were acquired using the Apo TIRF $60 \times$ oilimmersion objective (N.A. = 1.49) and Nikon C1si scanning confocal system. The sample was sequentially illuminated with a $405 \mathrm{~nm}$ laser for excitation of both studied nanoparticles and with a $488 \mathrm{~nm}$ laser for excitation of actin tagged with YFP in cells. For detection of signal from A-NDs, an emission band pass filter $450 \pm 15 \mathrm{~nm}$ was used, a band pass filter $515 \pm 15 \mathrm{~nm}$ was used for detection of actin tagged with YFP and a band pass filter $610 \mathrm{~nm} \pm 37.5 \mathrm{~nm}$ was used for detection of A-Si-NCs.

\section{Lactate dehydrogenase (LDH) viability/cytotoxicity test}

To estimate the influence of nanoparticles presence in the culture medium on the cell viability, the LDH viability/cytotoxicity test (Roche) was performed according to the manufacturer's protocol. The reaction consists of two phases. In the first phase $\mathrm{LDH}$ catalyzes the reduction of $\mathrm{NAD}+$ to $\mathrm{NADH}$ and $\mathrm{H}+$ by oxidation of lactate to pyruvate. In the second phase diaphorase uses $\mathrm{NADH}$ and $\mathrm{H}+$ to catalyze the reduction of a yellow coloured tetrazolium salt to dark red formazan. The optical density (OD) measured at $490 \mathrm{~nm}$ in cell lysate prepared from the living cells correlates with the amount of the living cells cultivated with the NPs, on the other hand, the OD at $490 \mathrm{~nm}$ measured in the cell supernatant correlates with the amount of ruptured dead cells that released the LDH into the medium. The value of
OD at wavelength $490 \mathrm{~nm}$ with reference wavelength at $600 \mathrm{~nm}$ was measured using an ELISA reader Synergy 2 (Biotek).

\section{Single nanoparticle spectra measurements}

Our purpose-built apparatus for the measurements of PL microspectra is designed for samples emitting low photon rate (like $\mathrm{Si}$ nanocrystals). It is based on an inverted microscope (IX-71, Olympus) equipped with an oil-immersion objective (UPlan FLN $100 \times / 1.35$ NA, Olympus). The sample is excited through the back-port of the microscope with a $405 \mathrm{~nm}$ continuous wave diode laser (Omicron) the excitation spot on the sample being around $40 \mu \mathrm{m}(88 \mu \mathrm{W})$. Sample image is projected onto the input slit of an imaging spectrometer (Acton SP-2300i, Princeton Instruments) and detected by a back-illuminated liquid nitrogen-cooled CCD camera (type Spec-10:400, Princeton Instruments). This enables us to record PL spectra of single emitting sources even when localized in a living cell. All measurements reported in this work were performed at room temperature.

\section{Results}

Physical characteristic of Si-NCs and ND: AFM, PL and zeta potential single Si-NCs studied in this work have size of 2.5$3 \mathrm{~nm}$ and the aggregates of interconnected Si nanocrystals (A-SiNCs) up to $150 \mathrm{~nm}$ (AFM see Fig. 1b, DLS). Photoluminescence (PL) maximum of Si-NCs with $\mathrm{SiO}_{2}$ shell with diameter of $2.5 \mathrm{~nm}$ is around $600 \mathrm{~nm}$ under excitation at $320 \mathrm{~nm}$ (see Fig. 1c), with photoluminescence (PL) lifetime found to be $\tau \sim 30 \mu \mathrm{s}$. The origin of the luminescence is discussed in ref. 34 and 35. Zeta potential measurements of A-Si-NCs colloidal suspension in distilled water showed that the particles are negatively charged $\zeta=-40 \pm 5 \mathrm{mV}$ (in agreement with ref. 36).

A-ND samples consist of a mixture of single NDs around $10 \mathrm{~nm}$ (see Fig. 1a) and of variously sized ND clusters up to about $460 \mathrm{~nm}$ in diameter (measured with DLS). The photoluminescence of A-NDs shows maximum in the UV and blue part of the visible spectrum showing no narrow peak (see Fig. 1). Zeta potential measurements of A-ND colloidal suspension in distilled water showed that the particles are positively charged $\zeta$ $=+25 \pm 5 \mathrm{mV}$.

The dependence of A-Si-NCs and A-ND zeta potential on $\mathrm{pH}$ is shown in Fig. 2a and b, respectively.

The relative height $\zeta$ of A-Si-NCs and A-NDs in water hinders the nanoparticles from penetrating the cell membrane; interestingly, the $\zeta$ of nanoparticles changed dramatically in nutrient media with or without fetal bovine serum (FBS) compared to water (see Table 1). For both A-Si-NCs and A-NDs in the nutrient medium the zeta potential had a Gaussian shape with full width at half maximum (FWHM) of $10 \mathrm{mV}$ (meaning that there are variously sized nanoparticles with different zeta potential values). The zeta potential of A-Si-NCs increased dramatically compared to the original value in water. On the other hand, the zeta potential of A-NDs decreased in nutrient media compared to water. This unexpected change of zeta potential to values close to $\zeta=0$ has an interesting consequence; the particle can 

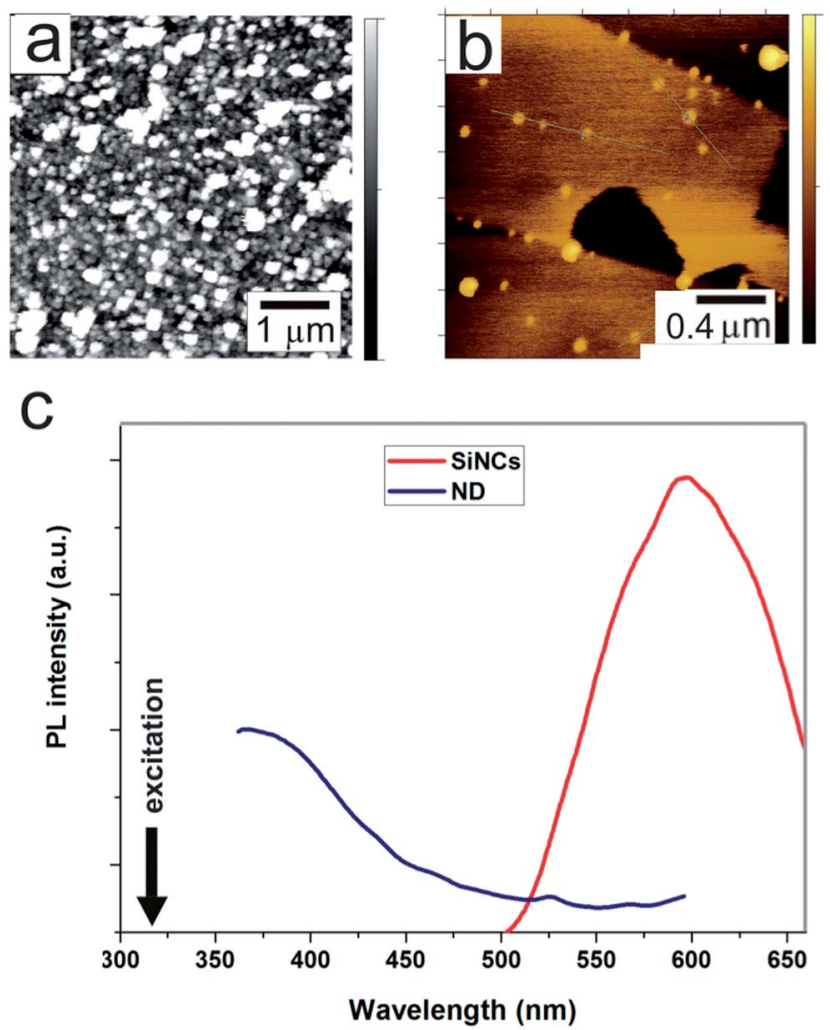

Fig. 1 Characterization of Si-NC and ND nanoparticles, AFM image of (a) A-NDs ( $Z$ scale $50 \mathrm{~nm}$ ) and (b) A-Si-NCs ( $Z$ scale $15.5 \mathrm{~nm}$ ) on highly ordered pyrolytic graphite, (c) PL spectra of A-Si-NCs (intensity divided by 10) and A-NDs in ethanol (excited at $320 \mathrm{~nm}$, measured with the FluoroMax-3 spectrometer, corrected for sensitivity of the apparatus and Raman scattering of solvent).

cross the cell membrane more easily than when having high zeta potential, both positive and negative. The origin of the zeta potential change is discussed later in this article.

\section{Interaction of $\mathrm{Si}-\mathrm{NCs}$ and ND with mammalian cells}

It is presumed that in a living organism accumulation and aggregation of nanoparticles can occur due to interaction with body fluids. This accumulation may enhance the overall cytotoxicity of nanoparticles and in order to simulate this phenomena variously sized agglomerates of nanoparticles in the cell studies were used.

In our previous experiments it was found that mammalian cells (HeLa, L929) incubated with Si-NCs (up to $8 \mu \mathrm{g} \mathrm{ml} \mathrm{m}^{-1}$ in nutrient media) can survive without significant difference in viability compared to control sample for at least 80 hours (longer times were not studied). ${ }^{37-39}$ According to growth curves (see ESI $\dagger$ ), ${ }^{39,40}$ for NDs (up to $8 \mu \mathrm{g} \mathrm{ml}^{-1}$ ) the number of live cells decreased by $30 \%$ compared to the control sample. In the case of A-NDs, the cells are not only sensitive to the dosage of the nanomaterial but also to time of A-ND addition to the cell culture. This decrease in cell viability was observed when cells were pre-seeded for $24 \mathrm{~h}$ and then the nanoparticles were added. However, when A-NDs were added together with freshly passaged cells, the number of live cells was even lower. These
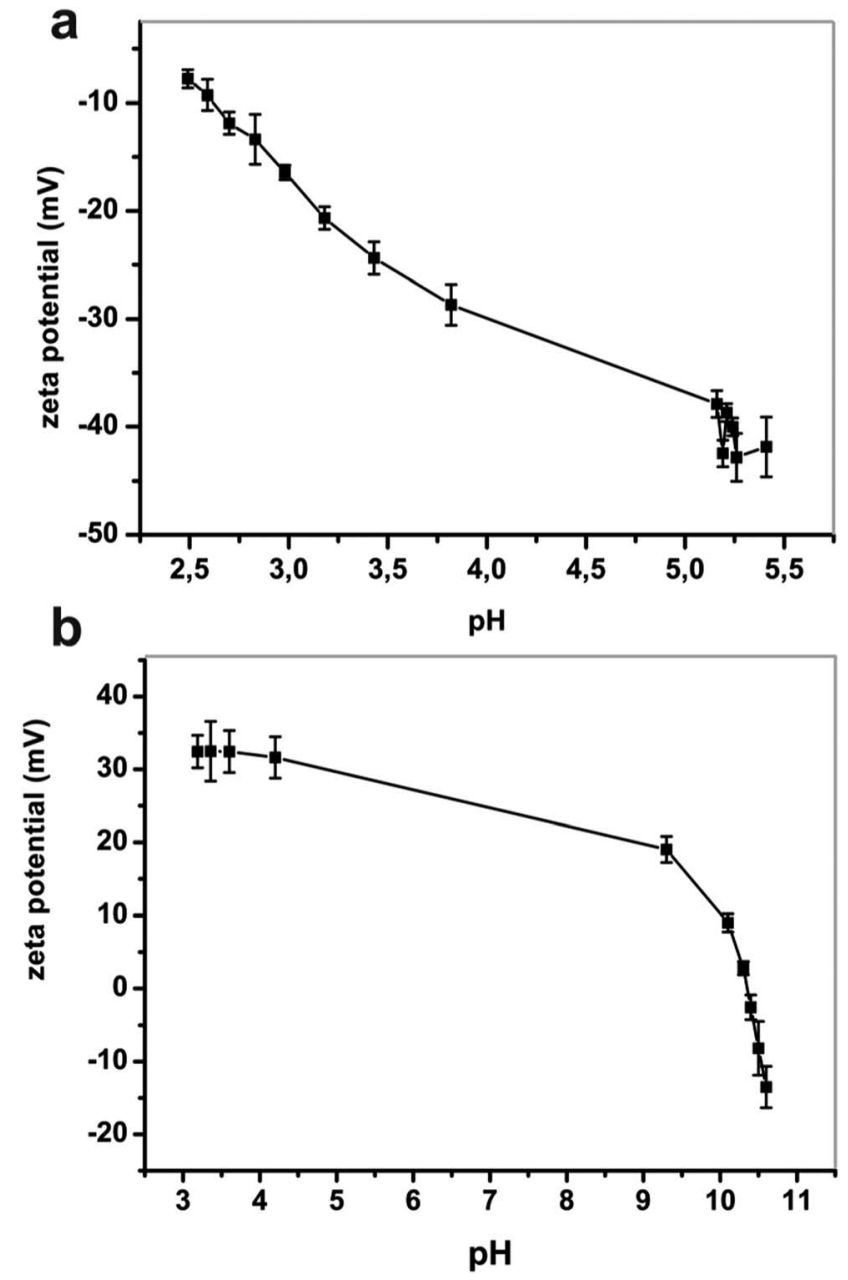

Fig. 2 Zeta potential dependence of (a) A-Si-NCs and (b) A-NDs on $\mathrm{pH}$ in water ( $\mathrm{pH}$ changed by titration using acids and bases).

Table 1 Zeta potential of A-Si-NCs and A-ND in water and McCoy's nutrient media with and without FBS

$\zeta[\mathrm{meV}]$

\begin{tabular}{lccc} 
Nanoparticle & $\begin{array}{r}\text { In water } \\
(\mathrm{pH} \text { 6.5) }\end{array}$ & $\begin{array}{l}\text { In nutrient medium } \\
\text { with FBS }(\mathrm{pH} 7.4)\end{array}$ & $\begin{array}{l}\text { In nutrient medium } \\
\text { without FBS }\end{array}$ \\
\hline A-Si-NCs & $-40 \pm 5$ & $+4 \pm 2$ & $+3 \pm 2$ \\
A-ND & $+25 \pm 5$ & $0 \pm 2$ & $0 \pm 2$
\end{tabular}

results have been also observed for time lapse microscopy performed for the same times and in the case of cells incubated with A-NDs, unusual active migration of cells (for details see ref. 40) was observed. When cells were incubated only with single NDs, no such effect was observed (see ESI $\dagger$ ). This significant difference in cell viability suggests that testing of nanomaterials should be in accordance with the final applications.

The reactions of A-NDs with cells was further studied with the help of SEM imaging. The cells incubated with A-NDs showed substantial changes in cell membrane. A-ND were attached to the outer cell membrane in huge aggregates 
interweaved with filaments (see Fig. 3) in contrast to a control sample and a sample incubated with Si-NCs.

\section{Cytotoxicity of Si-NCs and ND studied by LDH test}

In this part we are addressing how the interaction of live cells with Si-NCs and NDs affects the cell biochemistry and cytoskeleton. Mammalian cell line SAOS-2 was stably transfected with a vector expressing actin fused with fluorescent probe (YFP - Yellow Fluorescent Protein). As a result the actin structure of the transfected SAOS-2 is clearly visible under fluorescent microscope and enables us to observe the interaction with nanomaterials in real time (without any additional staining) in living cells. Moreover, a new test for cytotoxicity evaluation was performed and compared with our previous results and will be reported hereafter.

As a universal tool to study cytotoxicity, we have adopted lactase dehydrogenase ( $\mathrm{LDH})$ test which provides information about the status of a membrane and also indicates the level of cell damage as a whole. According to the $\mathrm{LDH}$ test within the first 24 hours, no significant difference was observed between the nanomaterial treated cells and the control cells (for all dosages used). The difference in viability of cells incubated with A-Si-NCs and A-NDs starts to be significant after 48 hours (Fig. 4). Significant decrease of the number of live cells was observed for dosages higher than $7.5 \mu \mathrm{g} \mathrm{ml}{ }^{-1}$. In the case of A-NDs, the number of live cells at the highest used dosage deceased to just $30 \%$, whereas in case of A-Si-NCs, $60 \%$ of the cells remained viable. The cells incubated with A-NDs started the apoptotic program (controlled cell death) during the first 24 hours, when the cell membrane was still intact. In next 24 hours of incubation, early signs of mild necrosis (uncontrolled cell death Fig. 5d) were observed, which is in agreement with membrane ruptures observed by LDH cytotoxicity test.

It should be mentioned that especially in case of Si-NCs, the overall cytotoxicity is strongly influenced by the nanoparticle surface. Our Si-NCs are covered by $\mathrm{SiO}_{2}$ shell (few atomic layers) which disables excitation energy transfer to singlet oxygen layer and inhibits production of measurable amount of reactive oxygen species (ROS). There are several studies on silicon nanocrystals with $\mathrm{Si}-\mathrm{H}$ surface passivation showing that these nanoparticles produce significant amounts of singlet oxygen and ROS under biological conditions and light excitation. ${ }^{\mathbf{4 1 , 4 2}}$

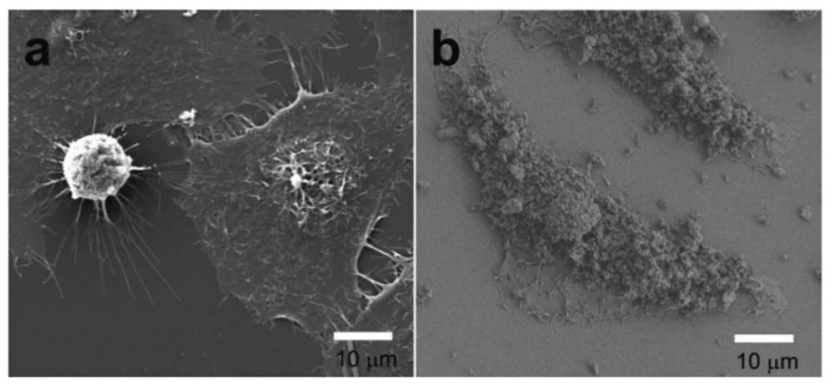

Fig. 3 SEM images of HeLa cells incubated with, (a) A-Si-NCs (cells have normal shape comparable to a control sample), (b) A-NDs (aggregates of ND interweaved with outer cell membrane actin structures (filopodia, microfilaments)).
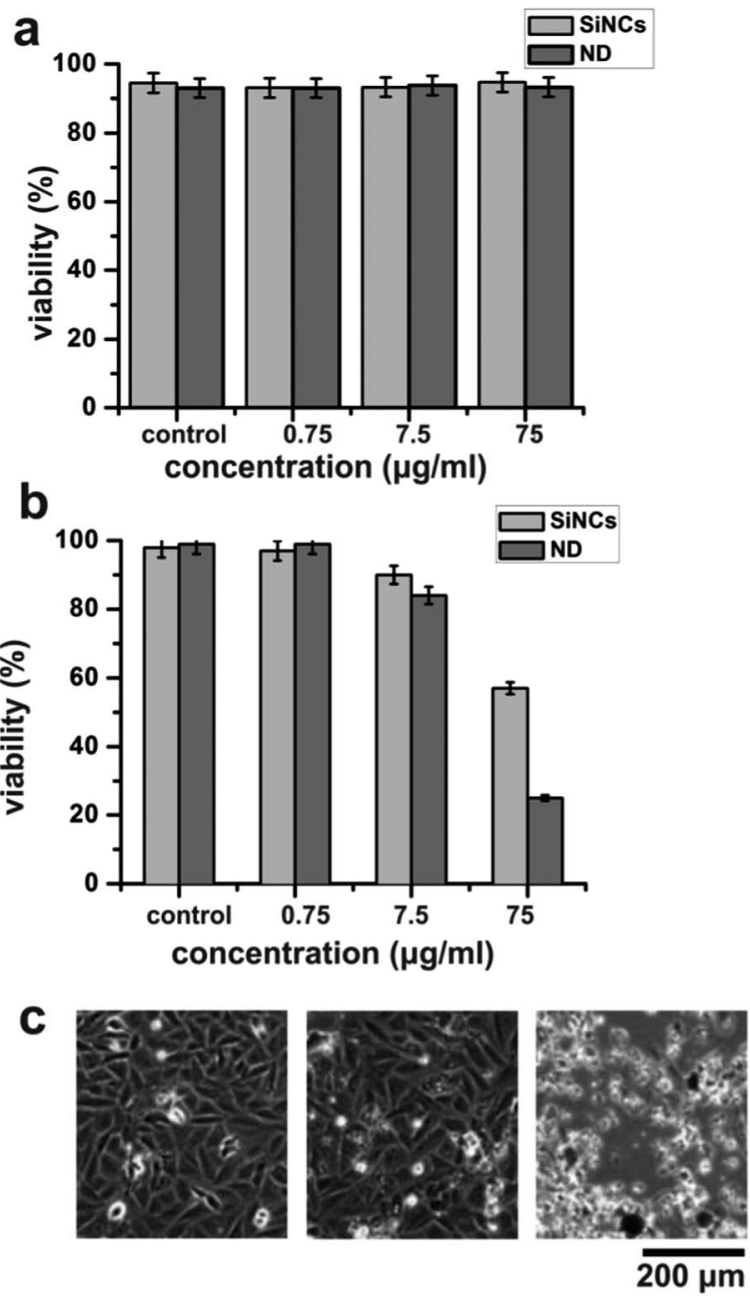

Fig. 4 Cytotoxicity of A-Si-NCs and A-NDs incubated with SAOS-2 cells for (a) 24 hours (b) 48 hours (lactase-dehydrogenase test), within the first 24 hours no significant difference between samples was observed, percentage of living cells shows higher cytotoxicity for higher dosages at 48 hours, (c) optical microscope images of SAOS-2 cells treated with $75 \mu \mathrm{g} \mathrm{ml}^{-1}$ (left to right: control, A-Si-NCs, A-NDs) for 48 hours.

These results reinforce the need for longer cytotoxicity studies of nanomaterials, especially since the negative properties of nanomaterials might not become apparent within the first 24 hours presence in bio-enviroment. ${ }^{43}$ The LDH cytotoxicity test results are in agreement with previous results obtained with more laborious and time consuming cytotoxicity test based on growth curves. ${ }^{37}$

\section{Cell cytoskeleton response to presence of Si-NCs and NDs}

Foreign nanomaterial inside the cell not only affects the chemistry of the cell but also the cytoskeleton responds to mechanical stress from the volume of the nanomaterial presented on/in the cell. The observation of cytoskeleton changes was carried out in this study on the mammalian cell line SAOS-2 (transfected by YEP), which enabled us to observe live interaction of nanomaterial with the actin structure of a cell. 

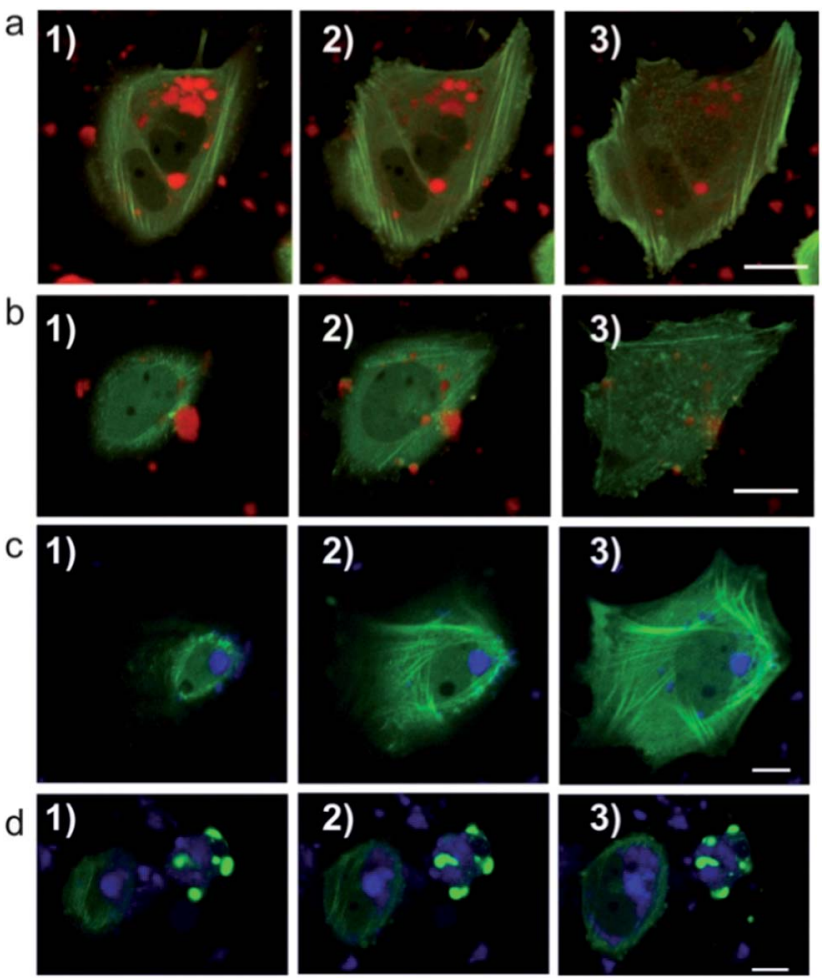

Fig. 5 Confocal fluorescence microscopy images of horizontal crosssections ( 1 - top section, 2 - middle section, 3 - bottom section of the cell) of live SAOS-2 cells incubated with nanomaterials $\left(75 \mu \mathrm{g} \mathrm{ml}^{-1}\right)$ (a) A-Si-NCs for 2 hours, (b) A-Si-NCs for 24 hours, (c) A-NDs for 2 hours, (d) A-NDs for 24 hours (the scale bar represents $10 \mu \mathrm{m}$ ). Color coding: green - actin, red - Si-NCs, blue - nanodiamonds. High resolution images are available in ESI. $\dagger$

The cells were plated 24 hours before the measurement, then the nanomaterial was added and samples were immediately observed under the microscope. The cell samples incubated with nanomaterial were studied for up to 28 hours. Two concentrations of A-NDs and A-Si-NCs were used $\left(7.5 \mu \mathrm{g} \mathrm{ml} \mathrm{ml}^{-1}\right.$ and $75 \mu \mathrm{g} \mathrm{ml}^{-1}$ in media). It was observed that for both nanomaterials, larger nanoparticles settled on the cell within the first 5-20 minutes as a result of gravitational forces. On the other hand, smaller particles were driven by Brownian motion and were observable in the medium all along the experiment. The aim of confocal microscopy was to study the dynamics and structure of the cell in the presence of nanomaterials. The smallest nanoparticles used in this study were observed using the wide-field PL micro-spectroscopy technique as shown below.

From resolution of the confocal images, the smallest aggregated nanoparticles observed had an estimated diameter of $\sim 150 \mathrm{~nm}$ in case of A-Si-NCs and $250 \mathrm{~nm}$ in case of A-NDs in live SAOS-2 cells (for A-NDs the resolution is lower due to autofluorescence of the cell in the same spectral area as the nanoparticles). The first A-Si-NCs appeared inside cells after 1 hour as revealed by examination of the $Z$-axis cross-sections of the $3 \mathrm{D}$ images of cells (for details see ESI $\dagger$ ). A-Si-NCs tend to accumulate in cells without causing significant damage to the cell cytoskeleton for all the nanoparticle concentrations used in this study (see Fig. 5a). For the highest dosage used, just small changes in actin structure were observed after 24 hour incubation with A-Si-NCs (see Fig. 5b). After this incubation, the cell volume occupied by the highest concentration of the A-Si-NCs made up to 1 percent (presuming that we were able to observe between 5 and $10 \%$ of all A-Si-NCs in the cell). 1 percent of cell volume corresponds to about 0.4 million of single Si-NC.

Similarly to A-Si-NCs, the first A-NDs were observed inside the cell after about 1 hour (see Fig. 5c). The A-NDs were concentrated perinucleary later on during the incubation. The cell volume occupied by the highest concentration of the A-NDs made up to 5 percent (we estimate that we were able to observe between 30 and $50 \%$ of all A-NDs in the cell) of the total cell volume after 24 hours (1 percent cell volume corresponds to about 5.000 of single ND). During the incubation, already after 2 hours changes in actin structures were observable for higher dosages of A-NDs as unorganized actin areas in close proximity of A-NDs. Nevertheless, after 24 hours significant changes were observable for all doses (see Fig. 5d). In the case of higher doses, the majority of cells were apoptotic and a high number of cell residua (necrosis) was observed.

In our confocal microscope study of live cells incubated with A-Si-NCs and A-NDs, we observe accumulation of nanoparticles in the perinuclear area, where lysosomes are localized as well. Based on real time confocal microscope observations it was concluded that the presumable way of A-Si-NCs and A-NDs entering the cell is endocytosis (in agreement with ref. 43). The scheme of suggested mechanism is shown in Fig. 7.

\section{Photoluminescence spectrum of single Si-NCs in live cell}

In biological experiments, it is crucial to have a tool to track reactions and objects on a molecular scale. Unlike somewhat problematic detection of single dye molecules which are strong and fast photo-bleaching, naturally fluorescent nanoparticles are better observable since they are more stable, non-bleaching and easier to be followed in single particle tracking. ${ }^{44}$

Our micro-spectroscopy set-up enables us to record emission spectra from a bare single Si-NC and a single ND deposited on a quartz substrate. Moreover, our set-up allows single spectra measurement on other substrates and in different environments. Even though in live cells such measurements are hampered by the cell autofluorescence, however, by using our purpose-built microscope, we were able to collect and spectrally resolve PL signal coming from single Si-NCs located inside a cell. Fig. 6 demonstrates benefits of Si-NCs as fluorescent markers over blue emitting NDs: the red color of Si-NCs luminescence contrasts sharply with green autofluorescence background (peaked around $500 \mathrm{~nm}$ ). This allows easy visual Si-NC observation as well as facile extraction of emission spectra. Panels $6 a$ and $6 c$ indicate the technique of recording the spectra. Panel $6 \mathrm{~b}$ represents emission spectra of single final Si-NCs nanoparticle spectra upon subtracting the background.

Emission spectra originating in single Si-NCs (or in small clusters composed of 5 single nanocrystals at most) located inside a living cell, have quite narrow emission spectrum compared with the ensemble spectrum in Fig. 1c (full width at 

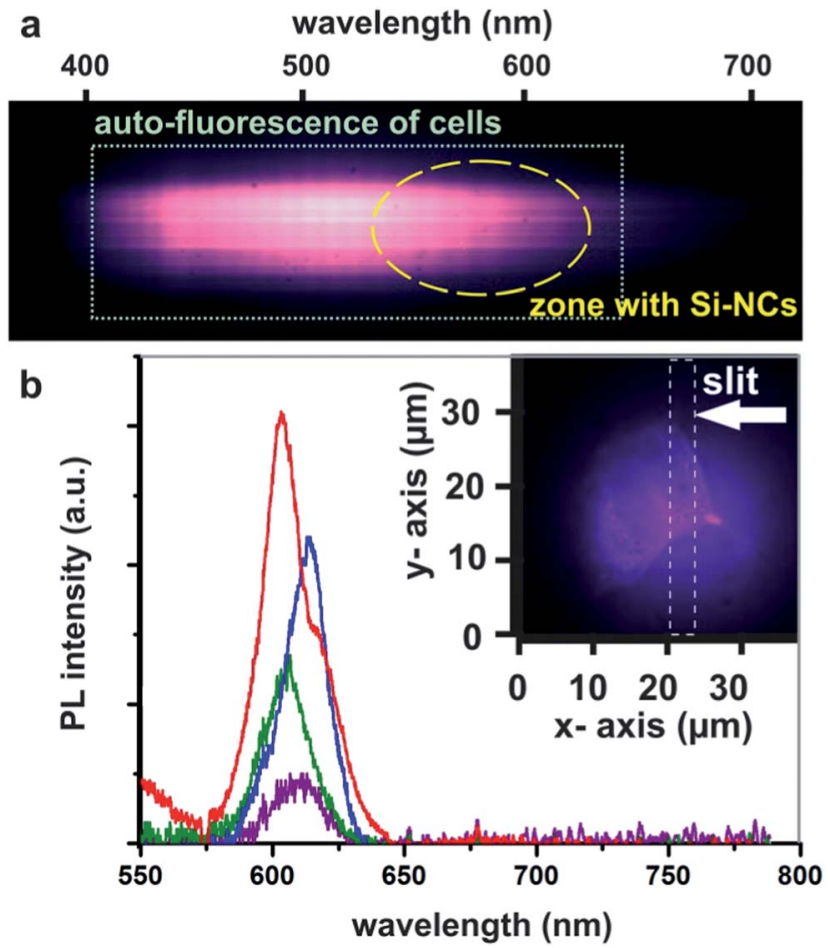

Fig. 6 Micro-spectroscopy of Si-NCs in live SAOS-2 cells, (a) PL spectral image of the slit-defined area of the cell ( $b$ inset) fluorescence image of SAOS-2 cell (excitation $405 \mathrm{~nm}$ ) with contours of the slit, defining the area from which PL spectra are taken. One can clearly see the broad cell-auto-fluorescence and also spectral zone where the SiNCs are emitting. (b) Emission spectra of several single Si-NCs upon subtracting the auto-fluorescence background.

half maximum 25-35 nm i.e. $80-120 \mathrm{meV}$ ) see Fig. $6 \mathrm{~b}$. Variations in their spectral position result from a relatively broad Si-NCs size distribution. Substantial narrowing of the distribution is needed in order to unveil possible impact of biological environment within the cell on the shape and position of the spectra. In particular, PL-mediated real-time information about chemical reactions involving the nanoparticle, or about nanoparticle possible size reduction (dissolution ${ }^{23}$ ), will be of great importance.

On the other hand, blue ND fluorescence blends to a large extent with the cell autofluorescence and can not be extracted (data not shown). If nitrogen-vacancy $(\mathrm{N}-\mathrm{V})$ center is present in NDs, they can be monitored via magnetic resonance in the cell ${ }^{45}$ and also optically. ${ }^{46}$

\section{Discussion}

Addressing the cytotoxicity of nanomaterials is a more complex task than of drugs. Although various scientific groups are producing nanomaterials by the same protocols, parameters such as surface groups and size vary significantly and this may lead to different behavior in biological environment. For this reason a more comprehensive characterization of nanomaterials is given in this study and behavior of nanomaterials in biological environment is explored by various methods.
The change of nanoparticles zeta potential in the presence of biological materials suggests significant surface changes. These changes can be explained by a nonspecific reaction of nanoparticles with components of nutrient medium such as bovine serum albumin, variously charged amino-acids, salts and vitamins. These components may bind to nanoparticle surface nonspecifically (the nature of chemical bonds was not the focus of this work) and as a result the measured zeta potential is not of the nanoparticle but of the nanoparticle embedded in biomolecular shell (sometimes called protein-corona ${ }^{47}$ ). The presence or absence of FBS in the nutrient medium had just a mild effect on zeta potential of particles as it is clear from the Table 1 (the shift of zeta potential maxima is just around $1 \mathrm{mV}$ ). A similar covering effect resulting in comparable change of zeta potential was observed for different nanoparticles with both negative and positive zeta potential. ${ }^{48}$

From the change of zeta-potential, confocal and SEM microscopy investigations we assume that the cell is not recognizing a bare nanoparticle but the protein/amino acid envelope ${ }^{48}$ which hides the entire nanoparticle. This is probably the reason for the increased nanoparticle uptake by the cells. Both A-Si-NCs and A-NDs tend to agglomerate in close proximity to the cell nucleus (in case of ND similar effect was observed) ${ }^{\mathbf{1 6 , 4 3}}$ as observed by confocal microscopy and schematically illustrated in Fig. 7. Based on real time confocal microscope observations it was concluded that the presumable way of A-SiNCs and A-NDs entering the cell is endocytosis (in agreement with ref. 43).

From the results of $\mathrm{LDH}$ test and confocal microscopy we can conclude, that within the first 24 hours the cells are negatively affected by the presence of A-NDs, apoptosis occurred and was followed by necrosis, as evidenced by ruptures of cell membranes detected for longer incubation times (one example of apoptotic cell is shown in Fig. 5d). The cytotoxicity of NDs seems to be influenced strongly by chemical groups on the

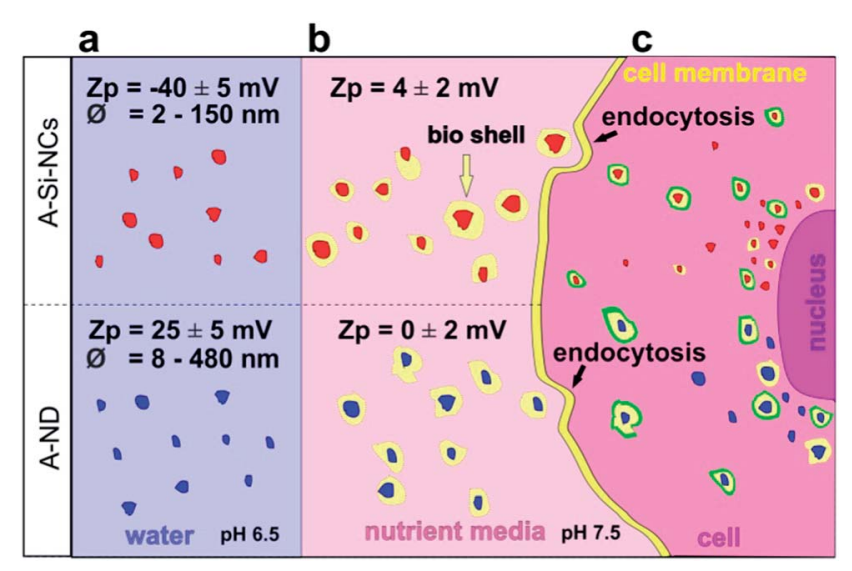

Fig. 7 Schematic representation of nanoparticles behavior in various solvents and their changes of $\zeta$ (zeta - potential) (a) nanoparticles in water $(\varnothing-$ diameter of particle), (b) nanoparticles in nutrient media covered with bio-molecular shell (possibly protein corona) which changes the overall zeta-potential (c) nanoparticles in the cell tend to agglomerate in close proximity of nucleus, the presumed way of entering the cell is endocytosis. 
surface. Change of the ND surface groups might diminish its negative effect on actin structure, for example Rezek et al. reported that ${ }^{49}$ the osteoblast cells preferred to grow on O-terminated diamonds surface and showed normal parameters but avoided H-terminated zones. The articles on NDs quite often provide contradictory cytotoxicity results. ${ }^{16,30}$ From current and previous studies on NDs, ${ }^{37,40}$ it is clear that the effect of accumulation of NDs enhances the overall cytotoxicity significantly (compared to toxicity single ND).

A-Si-NCs did not influence neither the cell biochemistry nor the cell cytotoxicity significantly as shown in our study. A decrease in cell viability was observed after 48 hours by LDH test (see Fig. 4) only for the highest dose used in this study. In all measurements A-Si-NCs showed better results than A-NDs. Recent in vivo studies show that accumulation of Si-NCs occurs in the case of mouse and monkey in liver and spleen ${ }^{26,27}$ and in case of mouse study ${ }^{27}$ inflammatory signs have been observed in addition.

The possibility of tracking Si-NCs spectrally is opening possibilities to detect size reduction of Si-NCs (dissolution ${ }^{23}$ ) in the cell and also we can obtain real-time information about chemical reactions involving the nanoparticles. ${ }^{40}$

\section{Conclusions}

In summary, we compared the effects of cytotoxicity and aggregation of light-emitting silicon nanocrystals and nanodiamonds inside live cells in particular in human osteoblasts and other mammalian cell lines. Both Si-NCs and NDs, as demonstrated by the presented study, are suitable for substituting the Cd-chalcogenide nanoparticles in biological applications. It turns out that the aggregation of silicon nanocrystals does not exhibit significant adverse influence on both the chemical and structural parameters of cells compared to NDs. This predestines the possible biological use of luminescent silicon nanocrystals to be directed towards living organisms or even towards humans. Aggregated nanodiamonds, on the other hand, show a mildly adverse effect on the cells in longterm studies. This observation indicates that the potential applications of nanodiamonds would be predominantly in vitro studies and the nanomaterial aggregation should be prevented as much as possible. In addition, for the first time we present photoluminescence spectra acquired from single Si nanocrystals embedded in a living cell. This enables, in the future application, to obtain not only the information about the position of nanoparticles, due to observance of change in PL spectra position and spectra shape, but also identify biological changes in proximity of nanoparticles.

\section{Acknowledgements}

This work was supported by grants: 202/09/H041, SVV-2010261304, PRVOUK-P24/LF1/3 (First Faculty of Medicine, Charles University, Prague), GAUK - 443211 (Faculty of Science UK, Prague), GACR P108/12/G108, GACR 202/09/2078 and European Regional Development Fund CZ.1.05/2.1.00/03.0076. The authors thanks to K. Kůsová (Inst. of Physics AS CR v. v. i.
Prague) for the DLS measurement, experimental assistance and advice, and P. Stepanek (Institute of Macromol. Chemistry AS CR, v.v.i. Prague) for zeta-potential measurements.

\section{Notes and references}

1 W. Yang, C. Shen, Q. Ji, H. An, J. Wang, Q. Liu and Z. Zhang, Nanotechnology, 2009, 20, 085102.

2 J. Weiss, P. Takhistov and J. McClements, J. Food Sci., 2006, 71(9), R107-R116.

3 L. T. Canham, Nanotechnology, 2007, 18, 185704.

4 A. I. Ekimov, Al. L. Efros and A. A. Onushchenko, Solid State Commun., 1985, 56(11), 921-924.

5 H. Mattoussi, J. M. Mauro, E. R. Goldman, T. M. Green, G. P. Anderson, V. C. Sundar and M. G. Bawendi, Phys. Status Solidi B, 2001, 224(1), 277-283.

6 F. Q. Chen and D. Gerion, Nano Lett., 2004, 4(10), 1827-1832.

7 L. Wang, D. K. Nagesha, S. Selvarasah, M. R. Dokmeci and R. L. Carrier, J. Nanobiotechnol., 2008, 6, 11.

8 T. Schwerdtle, F. Ebert, Ch. Thuy, C. Richter, L. H. F. Mullenders and A. Hartwig, Chem. Res. Toxicol., 2010, 23(2), 432-442.

9 L. T. Canham, Appl. Phys. Lett., 1990, 57, 1046-1048.

10 L. C. Qin, D. Zhou, A. R. Krauss and D. M. Gruen, Nanostruct. Mater., 1998, 10(I4), 649-660.

11 F. Erogbogbo, K. T. Yong, I. Roy, G. X. Xu, P. N. Prasad and M. T. Swihart, ACS Nano, 2008, 2(5), 873-878.

12 J. I. Chao, E. Perevedentseva, P.-H. Chung, K.-K. Liu, Ch.-Y. Cheng, Ch.-Ch. Chang and Ch.-L. Cheng, Biophys. J., 2007, 93(6), 2199-2208.

13 E. A. Ivukina, V. K. A. Sreenivasan, O. A. Stremovskiy, B. V. Veryugin, S. V. Lukash, A. V. Zvyagin, S. M. Deyev and R. V. Petrov, Dokl. Biochem. Biophys., 2011, 440(1), 231-233.

14 L. Marcon, F. Riquet, D. Vicogne, S. Szunerits, J. F. Bodart and R. Boukherrou, J. Mater. Chem., 2010, 20(37), 8064-8069.

15 A. M. Schrand, H. Juany, C. Carlson, J. J. Schlager, E. Osawa, S. M. Hussain and L. Dai, J. Phys. Chem. B, 2007, 111(1), 2-7.

16 L. P. McGuinness, Y. Yan, A. Stacey, D. A. Simpson, L. T. Hall, D. Maclaurin, S. Prawer, P. Mulvaney, J. Wrachtrup, F. Caruso, R. E. Scholten and L. C. Hollenberg, Nat. Nanotechnol., 2011, 6(6), 358-363.

17 V. N. Mochalin, O. Shenderova, D. Ho and Y. Gogotsi, Nat. Nanotechnol., 2012, 7, 11-23.

18 N. Mohan, C. S. Chen, H. H. Hsieh, Y. C. Wu and H. C. Chang, Nano Lett., 2010, 10(9), 3692-3699.

19 J. H. Park, L. Gu, G. von Maltzahn, E. Ruoslahti, S. N. Bhatia and M. J. Sailor, Nat. Mater., 2009, 8, 331-336.

20 S. P. Low, K. S. Williams, L. T. Canham and N. H. Voelcker, Biomaterials, 2006, 27(26), 4538-4546.

21 S. Quignard, G. Mosser, M. Boissière and T. Coradin, Biomaterials, 2012, 33(17), 4431-4442.

22 R. Jugdaohsingh, S. H. Anderson, K. L. Tucker, H. Elliott, D. P. Kiel, R. P. Thompson and J. J. Powell, Am. J. Clin. Nutr., 2002, 75, 887-893.

23 S. P. Low, N. H. Voelcker, L. T. Canham and K. A. Williams, Biomaterials, 2009, 30(15), 2873-2880. 
24 H. Nishimura, K. Ritchie, R. S. Kasai, M. Goto, N. Morone, H. Sugimura, K. Tanaka, I. Sase, A. Yoshimura, Y. Nakano, T. K. Fujiwara and A. Kusumi, J. Cell Biol., 2013, 202(6), 967-983.

25 R. Intartaglia, A. Barchanski, K. Bagga, A. Genovese, G. Das, P. Wagener, E. D. Fabrizio, A. Diaspro, F. Brandia and S. Barcikowski, Nanoscale, 2012, 4, 1271-1274.

26 S. Ivanov, S. Zhuravsky, G. Yukina, V. Tomson, D. Korolev and M. Galagudza, Materials, 2012, 5(10), 1873-1889.

27 J. Liu, F. Erogbogbo, K.-T. Yong, L. Ye, J. Liu, R. Hu, H. Chen, Y. Hu, Y. Yang, J. Yang, I. Roy, N. A. Karker, M. T. Swihart and P. N. Prasad, ACS Nano, 2013, 7(8), 7303-7310.

28 M. A. Dobrovolskaia, D. R. Germolec and J. L. Weaver, Nat. Nanotechnol., 2009, 4, 411-414.

29 Y.-R. Chang, H.-Y. Lee, K. Chen, Ch.-Ch. Chang, D.-S. Tsai, Ch.-Ch. Fu, T.-S. Lim, Y.-K. Tzeng, Ch.-Y. Fang, Ch.-Ch. Han, H.-Ch. Chang and W. Fann, Nat. Nanotechnol., 2008, 3, 284-288.

30 O. Faklaris, D. Garrot, V. Joshi, F. Druon, J. P. Boudou, T. Sauvage, P. Georges, P. A. Curmi and F. Treussart, Small, 2008, 4(12), 2236-2239.

31 K. Kůsová, O. Cibulka, K. Dohnalova, I. Pelant, J. Valenta, A. Fucikova, J. Lang, J. Englich, P. Matějka, P. Štěpánek and S. Bakardjieva, ACS Nano, 2010, 4(8), 4495-4504.

32 E. Osawa, Synthesis, properties and applications of ultracrystalline diamond, ed. D. M. Gruen, O. A. Shenderova and A. Ya. Vul', Springer, Dordrecht, The Nederlands, 2005, ch. 17.

33 E. D. Eidelman, V. I. Siklitsky, L. V. Sharonova, M. A. Yagovkina, A. Ya Vul', M. Takahashi, M. Inakuma, M. Ozawa and E. Ōsawa, Diamond Relat. Mater., 2005, 14, 1765-1769.

34 J. Valenta, A. Fucikova, I. Pelant, K. Kůsová, K. Dohnalová, A. Aleknavičius, O. Cibulka, A. Fojtík and G. Kada, New J. Phys., 2008, 10, 073022.

35 J. Valenta, A. Fucikova, F. Vácha, F. Adamec, J. Humpolíčková, M. Hof, I. Pelant, K. Kůsová,
K. Dohnalová and J. Linnros, Adv. Funct. Mater., 2008, 18, 2666-2672.

36 D. Mariotti, V. Švrček, J. W. J. Hamilton, M. Schmidt and M. Kondo, Adv. Funct. Mater., 2012, 22(5), 954-964.

37 A. Fucikova, J. Valenta, I. Pelant and V. Brezina, MRS Online Proc. Libr., 2009, 1241, 1241-XX03-11.

38 A. Fucikova, J. Valenta, I. Pelant, K. Kusova and V. Brezina, Phys. Status Solidi C, 2011, 8(3), 1093-1096.

39 A. Fucikova, J. Valenta, I. Pelant, V. Brezina and K. Kůsová, Chem. Pap., 2009, 63(6), 704-708.

40 A. Fucikova, Dissertation thesis: Bioapplications of novel nanostructured materials, Charles University in Prague 2012, https://www.is.cuni.cz/webapps/zzp/detail/44617/.

41 M. Fujii, N. Nishimura, H. Fumon, S. Hayashi, D. Kovalev, B. Goller and J. Diener, J. Appl. Phys., 2006, 100(12), 124302. 42 S. P. Low, K. A. Williams, L. T. Canham and N. H. Voelcker, J. Biomed. Mater. Res., Part A, 2010, 93(3), 1124-1131.

43 O. Faklaris, V. Joshi, T. Irinopoulou, P. Tauc, M. Sennour, H. Girard, C. Gesset, J. C. Arnault, A. Thorel, J. P. Boudou, P. A. Curmi and F. Treussart, ACS Nano, 2009, 3(12), 3955-3962. 44 J. H. Spille, T. Kaminski, H. P. Königshoven and U. Kubitscheck, Opt. Express., 2012, 20(18), 19697-19707.

45 C.-C. Fu, H.-Y. Lee, K. Chen, T.-S. Lim, H.-Y. Wu, P.-K. Lin, P.-K. Wei, P.-H. Tsao, H.-Ch. Chang and W. Fann, Proc. Natl. Acad. Sci. U. S. A., 2007, 104, 727-732.

46 Y. Yan, A. Stacey, D. A. Simpson, L. T. Hall, D. Maclaurin, S. Prawer, P. Mulvaney, J. Wrachtrup, F. Caruso, R. E. Scholten, L. C. L. Hollenberg, IQEC/CLEO Pacific Rim 2011, p. 601-602.

47 A. Salvati, A. S. Pitek, M. P. Monopoli, K. Prapainop, F. B. Bombelli, D. R. Hristov, P. M. Kelly, Christoffer Åberg, Eugene Mahon and K. A. Dawson, Nat. Nanotechnol., 2013, 8, 137-143.

48 S. Patila, A. Sandberg, E. Heckert, W. Self and S. Seal, Biomaterials, 2007, 28(31), 4600-4607.

49 B. Rezek, L. Michalíková, E. Ukraintsev, A. Kromka and M. Kalbacova, Sensors, 2009, 9(5), 3549-3562. 